Louisiana State University

LSU Digital Commons

Faculty Publications

Department of Mathematics

6-29-2017

\title{
Stability and robustness analysis for a multi-species chemostat model with uncertainties
}

\author{
Frederic Mazenc \\ Laboratoire des Signaux et Systèmes \\ Michael Malisoff \\ Louisiana State University \\ Gonzalo Robledo \\ Universidad de Chile
}

Follow this and additional works at: https://digitalcommons.Isu.edu/mathematics_pubs

\section{Recommended Citation}

Mazenc, F., Malisoff, M., \& Robledo, G. (2017). Stability and robustness analysis for a multi-species chemostat model with uncertainties. Proceedings of the American Control Conference, 2130-2134. https://doi.org/10.23919/ACC.2017.7963267

This Conference Proceeding is brought to you for free and open access by the Department of Mathematics at LSU Digital Commons. It has been accepted for inclusion in Faculty Publications by an authorized administrator of LSU Digital Commons. For more information, please contact ir@lsu.edu. 


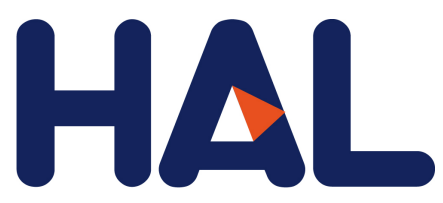

archives-ouvertes

\title{
Stability and Robustness Analysis for a Multi-Species Chemostat Model with Uncertainties
}

\author{
Frédéric Mazenc, Michael Malisoff, Gonzalo Robledo
}

\section{To cite this version:}

Frédéric Mazenc, Michael Malisoff, Gonzalo Robledo. Stability and Robustness Analysis for a MultiSpecies Chemostat Model with Uncertainties. IEEE American Control Conference (ACC) 2017, May 2017, Seattle, United States. pp.1-5, 10.23919/ACC.2017.7963267 . hal-01660127

HAL Id: hal-01660127

https://hal.inria.fr/hal-01660127

Submitted on 10 Dec 2017

HAL is a multi-disciplinary open access archive for the deposit and dissemination of scientific research documents, whether they are published or not. The documents may come from teaching and research institutions in France or abroad, or from public or private research centers.
L'archive ouverte pluridisciplinaire HAL, est destinée au dépôt et à la diffusion de documents scientifiques de niveau recherche, publiés ou non, émanant des établissements d'enseignement et de recherche français ou étrangers, des laboratoires publics ou privés. 


\section{Stability and Robustness Analysis for a Multi- Species Chemostat Model with Uncertainties}

Frédéric Mazenc
Michael Malisoff

Gonzalo Robledo

\begin{abstract}
We prove stability and robustness results for chemostat models with one substrate, an arbitrary number of species, a constant dilution rate, and constant inputs of the species. Unlike all previous works, we prove input-tostate stability under uncertainties in important cases where the controls are the input nutrient concentration and the species inputs. Our assumptions ensure global asymptotic stability for an equilibrium, which can allow persistence of multiple species, when the uncertainties are zero. We allow arbitrarily large bounds on the uncertainties in the species dynamics, and equilibria that can be in the boundary of the state space.
\end{abstract}

Index Terms-Bioreactors, nonlinear, stability, robustness

\section{INTRODUCTION}

The chemostat is a mathematical model and a laboratory device that is used for the continuous culture of microorganisms. Since its introduction in [14] and [16], it has been studied extensively, because of its vital role in ecology and microbiology as an ideal representation of microorganism growth, natural environments such as lakes, and wastewater treatment processes [1]. It is used in industrial applications that are of compelling engineering interest. See [18].

This has motivated our ongoing work (begun in [2], [3], [8], [9], [10], [11], [12], [13], and [17]) on methods to ensure desired asymptotic behaviors in chemostats, including the coexistence of multiple competing species, convergence to equilibria, or delay compensation. As noted in [18], the classical model of competition in the chemostat is

$$
\left\{\begin{aligned}
\dot{s}(t) & =D\left[s_{\mathrm{in}}-s(t)\right]-\sum_{i=1}^{n} Y_{i}^{-1} \mu_{i}(s(t)) x_{i}(t) \\
\dot{x}_{i}(t) & =x_{i}(t) \mu_{i}(s(t))-D x_{i}(t), i=1, \ldots, n
\end{aligned}\right.
$$

where $n \geq 2$ microbial species (with concentrations $\left.x_{1}, \ldots, x_{n}\right)$ compete for a nutrient with concentration $s$. The positive constants $D$ and $s_{\text {in }}$ are the dilution rate and input nutrient concentration, respectively, and $Y_{i}$ is a positive yield constant related to the conversion of the substrate into biomass for each $i$. The $\mu_{i}$ 's for $i=1, \ldots, n$ are strictly increasing, satisfy $\mu_{i}(0)=0$, and are assumed to be continuously differentiable; they describe the consumption of

Partially supported by the Regional Program MATH-AmSud STADE (Mazenc and Robledo) and by NSF Grant 1408295 (Malisoff).

Mazenc is with EPI DISCO Inria-Saclay, Laboratoire des Signaux et Systèmes (L2S, UMR CNRS 8506), CNRS, CentraleSupélec, Université Paris-Sud, 3 rue Joliot Curie, 91192, Gif-sur-Yvette, France, frederic.m azencel2s.centralesupelec.fr.

Malisoff is with Department of Mathematics, 303 Lockett Hall, Louisiana State University, Baton Rouge, LA 70803-4918, USA, mali soff@lsu.e du.

Robledo is with Departamento de Matemáticas, Universidad de Chile, Casilla 653, Santiago, Chile, grobledo@uchile.cl. the nutrient by species $i$. The model assumes that the growth of species $i$ is proportional to the nutrient consumption.

Well known results (e.g., [4] and [18]) imply that if the preceding conditions hold and $0<\mu_{n}^{-1}(D)<\mu_{n-1}^{-1}(D)<$ $\ldots<\mu_{2}^{-1}(D)<\mu_{1}^{-1}(D)<s_{\text {in }}$, then the following competitive exclusion principle holds: $\lim _{t \rightarrow+\infty} s(t)=\mu_{n}^{-1}(D)$, $\lim _{t \rightarrow+\infty} x_{n}(t)=Y_{n}\left[s_{\text {in }}-\mu_{n}^{-1}(D)\right]$, and $\lim _{t \rightarrow+\infty} x_{i}(t)=$ 0 if $1 \leq i \leq n-1$. The preceding conditions imply that only the $n$th species persists, because it only needs the lowest nutrient concentration.

However, multiple species are often observed to persist in chemostats with one substrate, which motivated many works on ways to explain coexistence in chemostats. There are several approaches to explaining coexistence in bioreactor models, including models whose qualitative behavior and analytical treatment are considerably more complex than (1) and we refer to [2], [5], [7], [8], [11], [13], [15], and [19] without claiming completeness. Nevertheless, we will focus our attention on the approach from [17], which introduces a model with constant inputs $x_{i}^{0} \geq 0$ of the $i$ th competing species (for $i=1, \ldots, n$ ) described by the system

$$
\left\{\begin{aligned}
\dot{s}(t) & =D\left[s_{\text {in }}-s(t)\right]-\sum_{i=1}^{n} Y_{i}^{-1} \mu_{i}(s(t)) x_{i}(t) \\
\dot{x}_{i}(t) & =x_{i}(t) \mu_{i}(s(t))+D\left[x_{i}^{0}-x_{i}(t)\right], 1 \leq i \leq n .
\end{aligned}\right.
$$

In [17], the authors obtained sufficient conditions ensuring the coexistence of multiple species. The work [17] used polytopic Lyapunov functions, which were also used in [3].

However, it is well known that chemostats can contain uncertainties (e.g., unmodeled features, or uncertainties in the input concentrations, which are common in applications). Therefore, an even more accurate model than (2) is

$$
\left\{\begin{aligned}
\dot{s}(t)= & D\left[s_{\mathrm{in}}-s(t)\right]-\sum_{i=1}^{n} \mu_{i}(s(t)) x_{i}(t)+\delta_{0}(t) \\
\dot{x}_{i}(t)= & x_{i}(t) \mu_{i}(s(t)) \\
& +D\left[x_{i}^{0}-x_{i}(t)\right]+\delta_{i}(t), 1 \leq i \leq n,
\end{aligned}\right.
$$

where the $\mu_{i}$ 's are as before, and the unknown measurable essentially bounded functions $\delta_{i}:[0,+\infty) \rightarrow\left[\underline{d}_{i}, \bar{d}_{i}\right]$ for $i=0,1, \ldots, n$ represent uncertainties and have known upper and lower bounds $\bar{d}_{i}$ and $\underline{d}_{i}$, respectively, and where we used a change of coordinates (based on a scaling of the $x_{i}$ 's and $x_{i}^{0}$ 's) to remove the $Y_{i}$ 's. Two of our assumptions will be that $\underline{d}_{0}>-D s_{\text {in }}$ and $\underline{d}_{i} \geq-D x_{i}^{0}$ for $i=1,2, \ldots, n$; see Section II for our assumptions. Therefore, all solutions of (3) with initial states $(s(0), x(0))$ in $\mathcal{X}=(0,+\infty)^{n+1}$ remain in $\mathcal{X}$ for all $t \geq 0$, so (3) has the state space $\mathcal{X}$, and (3) will be the subject of this paper. 
In the next section, we provide our theorem for (3), which uses $s_{\text {in }}$ and the $x_{i}^{0}$ 's as controls. Our work is novel in its use of the model (3), which we believe has not been studied in the presence of nonzero uncertainties. Our new Lyapunov construction is the key ingredient for proving valuable inputto-state stability (ISS) robustness properties, which cannot be deduced from the polytopic Lyapunov functions from [17]. Also, the equilibria that we stabilize are in the boundary of $\mathcal{X}$ when at least one $x_{i}^{0}$ is 0 , so the present paper covers a broad class of equilibria. Hence, this work provides a new theoretical result with valuable implications for future real time applications to bioprocess engineering.

\section{Assumptions, Definitions, And Main Result}

We will prove ISS properties for the dynamics for the error $\mathcal{E}(t)=\left(s(t)-s_{*}, x(t)-x_{*}\right)$ with respect to the disturbance vector $\delta=\left(\delta_{0}, \delta_{1}, \ldots, \delta_{n}\right)$, for a large class of possible equilibrium points $\mathcal{E}_{*}=\left(s_{*}, x_{*}\right)$, where $(s, x)$ is the state of (3), $x_{*}=\left(x_{1 *}, \ldots, x_{n *}\right)$, and $x=\left(x_{1}, \ldots, x_{n}\right)$. The ISS framework is used extensively in engineering; see [6] for ISS for systems without state constraints. To allow state constraints, we use a variant of the usual ISS property.

To explain this variant, we first need two definitions. Let $\mathcal{K}_{\infty}$ be the set of all continuous strictly increasing unbounded functions $\gamma:[0,+\infty) \rightarrow[0,+\infty)$ such that $\gamma(0)=0$; and let $\mathcal{K} \mathcal{L}$ be the set of all continuous functions $\bar{\beta}:[0,+\infty) \times[0,+\infty) \rightarrow[0,+\infty)$ such that (i) for each $t \geq 0$, the function $f(s)=\bar{\beta}(s, t)$ is of class $\mathcal{K}_{\infty}$ and (ii) for each $s \geq 0$, the function $g(t)=\bar{\beta}(s, t)$ is nonincreasing and satisfies $\lim _{t \rightarrow+\infty} g(t)=0$. By ISS of a system of the form $\dot{\mathcal{E}}(t)=\mathcal{F}(\mathcal{E}(t), \delta(t))$ with respect to a pair $(\mathcal{D}, \mathcal{S})$, we mean that there exist $\beta \in \mathcal{K} \mathcal{L}$ and $\gamma \in \mathcal{K}_{\infty}$ such that $|\mathcal{E}(t)| \leq \beta(|\mathcal{E}(0)|, t)+\gamma\left(|\delta|_{[0, t]}\right)$ holds for all $t \geq 0$, all solutions $\mathcal{E}(t)$ of this system that have initial states $\mathcal{E}(0) \in \mathcal{S}$, and all measurable essentially bounded functions $\delta:[0,+\infty) \rightarrow \mathcal{D}$. Here and in the sequel, $|\cdot|$ is the Euclidean norm, and $|\cdot|_{[0, t]}$ (resp., $|\cdot|_{\infty}$ ) is the essential supremum over $[0, t]$ for all $t \geq 0$ (resp., over $[0,+\infty)$ ). Assume:

Assumption 1: The $\mu_{i}$ 's in (3) have the Monod form

$$
\mu_{i}(s)=\frac{m_{i} s}{a_{i}+s} \text { for } i=1,2, \ldots, n,
$$

where the $m_{i}$ 's and $a_{i}$ 's are known positive constants.

Assumption 2: The constants $s_{*}>0$ and $s_{\text {in }}$ are such that

$$
\begin{aligned}
& \mu_{i}\left(s_{*}\right)<D \text { for } i=1,2, \ldots, n \\
& \text { and } s_{\text {in }}=s_{*}+\sum_{i=1}^{n} \frac{\mu_{i}\left(s_{*}\right) x_{i}^{0}}{D-\mu_{i}\left(s_{*}\right)},
\end{aligned}
$$

the $x_{i}^{0}$ 's are nonnegative constants, and $0<D<\mu_{n}\left(s_{\text {in }}\right)$. $\square$

From Assumption 2, we obtain $\mu_{n}\left(s_{*}\right)<\mu_{n}\left(s_{\text {in }}\right)$, so since $\mu_{n}$ is strictly increasing, it follows that $s_{*} \in\left(0, s_{\text {in }}\right)$. We can always satisfy Assumption 2 for all constants $D \in\left(0, m_{n}\right)$, by first fixing $s_{*}>0$ such that $\mu_{i}\left(s_{*}\right)<D$ for all $i$, and then picking the $x_{i}^{0}$ 's to be large enough so that $D<\mu_{n}\left(s_{\text {in }}\right)$ and $s_{\text {in }}>0$, i.e., we view $s_{\text {in }}$ as a constant control. By the symmetry of the system (3) in its components $x_{i}$, we can replace the condition $D<\mu_{n}\left(s_{\text {in }}\right)$ by the condition that $D<\mu_{i}\left(s_{\text {in }}\right)$ for any $i$, by renumbering the species. By
Assumption 2, it follows that when the $\delta_{i}$ 's in (3) are 0, the system (3) has the equilibrium $\mathcal{E}_{*}=\left(s_{*}, x_{*}\right)$, where

$$
x_{i *}=\frac{D x_{i}^{0}}{D-\mu_{i}\left(s_{*}\right)} \text { for } i=1, \ldots, n .
$$

We can allow many $x_{i *}$ 's through different choices of $x_{i}^{0}$, s, i.e., we also use the $x_{i}^{0}$ 's as controls. Since the $x_{i}^{0}$ 's are nonnegative, we have $\mathcal{E}_{*} \in[0,+\infty)^{n+1}$; and $\mathcal{E}_{*} \in(0,+\infty)^{n+1}$ when the $x_{i}^{0}$ 's are all positive. Our assumptions on the unknown measurable essentially bounded functions $\delta_{i}$ in (3) are as follows, where $\mathcal{P}=\left\{i \in\{1,2, \ldots, n\}: x_{i}^{0}>0\right\}$ :

Assumption 3: We have $\delta(t) \in\left[\underline{d}_{0}, \bar{d}_{0}\right] \times \ldots \times\left[\underline{d}_{n}, \bar{d}_{n}\right]$ for almost all $t \geq 0$, where the known constants $\underline{d}_{i}$ and $\bar{d}_{i} \geq 0$ are such that $D s_{\text {in }}+\underline{d}_{0}>0, \bar{d}_{0}<0.5 D s_{*}$, and $D x_{i}^{0}+\underline{d}_{i}>0$ for all $i \in \mathcal{P}$, and $\underline{d}_{i}=0$ for all $i \in\{1,2, \ldots, n\} \backslash \mathcal{P}$.

We can prove the following result, where $(s(t), x(t))$ is the state of (3):

Theorem 1: If Assumptions 1-3 hold, then for all constants $\underline{x}>0$ and $\bar{s} \geq s_{\text {in }}$, the dynamics for the error vector $\mathcal{E}=(s, x)-\mathcal{E}_{*}$ have the ISS property with respect to $(\mathcal{D}, \mathcal{S})$ with the disturbance set $\mathcal{D}=\left[\underline{d}_{0}, \bar{d}_{0}\right] \times \ldots \times\left[\underline{d}_{n}, \bar{d}_{n}\right]$ and $\mathcal{S}=\left\{\mathcal{E}: \mathcal{E}+\mathcal{E}_{*} \in(0, \bar{s}] \times(0,+\infty)^{n-1} \times(\underline{x},+\infty)\right\}$.

Before discussing our proof, we make several remarks about the novelty and value of our theorem.

Remark 1: Our choice of $\mathcal{S}$ in Theorem 1 corresponds to the requirement that $(s(0), x(0)) \in(0, \bar{s}] \times(0,+\infty)^{n-1} \times$ $(\underline{x},+\infty)$. However, since $\bar{s} \geq s_{\text {in }}$ and $\bar{x}>0$ are arbitrary, we conclude that when the $\delta_{i}$ 's are zero, all solutions $(s(t), x(t))$ of (3) starting in $\mathcal{X}=(0,+\infty)^{n+1}$ remain in $\mathcal{X}$ at all positive times and satisfy $\lim _{t \rightarrow+\infty}(s(t), x(t))=\left(s_{*}, x_{*}\right)$. This ensures persistence of the $i$ th species for all $i \in \mathcal{P}$ (and $\lim _{t \rightarrow+\infty} x_{i}(t)=0$ for all $\left.i \in\{1, \ldots, n\} \backslash \mathcal{P}\right)$.

Remark 2: We do not restrict the values of $\bar{d}_{i} \geq 0$ for $i \geq 1$, so Theorem 1 ensures ISS under arbitrarily large sup norms on the $\delta_{i}$ 's for $i \geq 1$. A key ingredient in our proof is a Lyapunov-like function $V$ and a function $T_{3} \in \mathcal{K}_{\infty}$ such that $V$ satisfies the usual Lyapunov positive definiteness and decay conditions along all solutions $\mathcal{E}(t)$ of the error dynamics for all $t \geq T_{3}(|\mathcal{E}(0)|)$. Using $V$ instead of the polytopic Lyapunov functions from [17] allows us to prove key ISS results that were beyond the scope of [17].

\section{SKetCh OF PROOF OF TheOREM 1}

\section{A. Preliminary State Bounds}

Since (3) is forward complete on $\mathcal{X}=(0,+\infty)^{n+1}$, we can first fix any solution $(s(t), x(t))$ of (3) all of whose components are positive for all $t \geq 0$ for which $\mathcal{E}(0) \in \mathcal{S}$. Set $\bar{s}^{\sharp}=\bar{s}+\left(\bar{d}_{0} / D\right)$ and

$$
(\tilde{s}, \tilde{x})=\left(s-s_{*}, x-x_{*}\right) .
$$

Then Assumption 3 implies that $s(t) \leq \bar{s}^{\sharp}$ for all $t \geq 0$. We next produce functions $T_{i} \in \mathcal{K}_{\infty}$ for $i=1,2,3$, whose class $\mathcal{K}_{\infty}$ properties will be used later to build an ISS estimate that is valid for all times $t \geq 0$, using three lemmas. Our first lemma is:

Lemma 1: If Assumptions 1-3 hold, then there is a $T_{1} \in$ $\mathcal{K}_{\infty}$ such that $s(t) \leq s_{\text {in }}+\left(\bar{d}_{0} / D\right)$ for all $t \geq T_{1}(|\tilde{s}(0)|)$. $\square$ 
Proof: (Sketch.) If there is a $t_{l} \geq 0$ such that $s\left(t_{l}\right) \leq$ $s_{\text {in }}+\left(\bar{d}_{0} / D\right)$, then for all $t>t_{l}$, we have $s(t) \leq s_{\text {in }}+$ $\left(\bar{d}_{0} / D\right)$. Next, consider the case where $s(0)>s_{\text {in }}+\left(\bar{d}_{0} / D\right)$. Consider any $t \geq 0$ such that $\min _{\ell \in[0, t]} s(\ell)>s_{\text {in }}+\left(\bar{d}_{0} / D\right)$. For any such $t$, we also have $\max _{\ell \in[0, t]} \dot{s}(\ell)<0$. Then since $D<\mu_{n}\left(s_{\text {in }}\right)$, and since $\mu_{n}$ is nondecreasing and $D x_{n}^{0}+\underline{d}_{n} \geq$ 0 and $s_{*}<s_{\text {in }} \leq s(\ell)$ for all $\ell \in[0, t]$, we deduce from (3) that $\dot{x}_{n}(\ell) \geq 0$ and so also $x_{n}(\ell) \geq x_{n}(0) \geq \underline{x}$ for all $\ell \in[0, t]$, and $\dot{s}(\ell) \leq-\mu_{n}(s(\ell)) x_{n}(\ell)$ for all $\ell \in[0, t]$, so

$$
\begin{aligned}
t \mu_{n}\left(s_{\text {in }}\right) \underline{x} & \leq \int_{0}^{t} \mu_{n}\left(s_{\text {in }}\right) x_{n}(\ell) \mathrm{d} \ell \\
& \leq s(0)-s(t) \leq s(0)-s_{*} \leq|\tilde{s}(0)| .
\end{aligned}
$$

Hence, $t \leq|\tilde{s}(0)| /\left(\mu_{n}\left(s_{\text {in }}\right) \underline{x}\right)$, so there is a $t_{*} \in$ $\left[0,2|\tilde{s}(0)| /\left(\mu_{n}\left(s_{\text {in }}\right) \underline{x}\right)\right]$ such that $s\left(t_{*}\right) \leq s_{\text {in }}+\left(\bar{d}_{0} / D\right)$. Hence, we can choose $T_{1}(r)=2 r /\left(\mu_{n}\left(s_{\text {in }}\right) \underline{x}\right)$.

Set $\sigma(t)=s(t)+x_{1}(t)+\ldots+x_{n}(t)$ for all $t \geq 0$ and

$$
C=2\left(s_{\text {in }}+\sum_{i=1}^{n} x_{i}^{0}+\frac{1}{D} \sum_{i=0}^{n} \bar{d}_{i}\right)
$$

and fix any constant $\lambda_{1}>1$. We prove:

Lemma 2: If Assumptions 1-3 hold, then there is a $T_{2} \in$ $\mathcal{K}_{\infty}$ such that $\sigma(t) \leq \lambda_{1} C$ holds for all $t \geq T_{2}(|\mathcal{E}(0)|)$.

Proof: (Sketch.) By (3), we have $\dot{\sigma}(t) \leq 0.5 C D-$ $D \sigma(t)$ for all $t \geq 0$. It follows that $\sigma(t) \leq u(t)$ for all $t \geq 0$, where $u$ is the solution of the initial value problem

$$
\dot{u}(t)=\frac{C D}{2}-\frac{1}{2} D u(t), u(0)=\sigma(0) .
$$

Since $\lambda_{1}>1$, we deduce that $\sigma(t) \leq u(t) \leq \lambda_{1} C$ for all $t \geq 0$ if $\sigma(0) \in\left(0, \lambda_{1} C\right]$. Hence, there exists a function $T_{2}^{b} \in \mathcal{K}_{\infty}$ such that $\sigma(t) \leq \lambda_{1} C$ for all $t \geq T_{2}^{b}(\sigma(0))$ when $\sigma(0)>\lambda_{1} C$. Therefore, the lemma will follow once we choose a function $T_{2} \in \mathcal{K}_{\infty}$ such that $T_{2}(|\mathcal{E}(0)|) \geq T_{2}^{b}(\sigma(0))$ holds for all solutions of (3) such that $\sigma(0)>\lambda_{1} C$.

To find $T_{2}$, first note that our formulas for $\sigma$ and $s_{\text {in }}$ give

$$
\begin{aligned}
\sigma(0) & =\tilde{s}(0)+\sum_{i=1}^{n} \tilde{x}_{i}(0)+s_{*}+\sum_{i=1}^{n} \frac{D x_{i}^{0}}{D-\mu_{i}\left(s_{*}\right)} \\
& \leq|\tilde{s}(0)|+\sum_{i=1}^{n}\left|\tilde{x}_{i}(0)\right|+s_{\text {in }}+\sum_{i=1}^{n} x_{i}^{0} .
\end{aligned}
$$

We next consider two cases. Case 1: If $|\mathcal{E}(0)| \leq\left(s_{\text {in }}+\right.$ $\left.x_{1}^{0}+\ldots+x_{n}^{0}\right) /(n+1)$, then the inequality in (11) gives $\sigma(0) \leq \lambda_{1} C$, so this case does not produce any restriction on the allowable values of $T_{2}$. Case 2: If $|\mathcal{E}(0)|>\left(s_{\text {in }}+\right.$ $\left.x_{1}^{0}+\ldots+x_{n}^{0}\right) /(n+1)$, then we use the fact that (11) gives $T_{2}^{\mathrm{b}}\left((n+1)|\mathcal{E}(0)|+s_{\text {in }}+x_{1}^{0}+\ldots+x_{n}^{0}\right) \geq T_{2}^{\mathrm{b}}(\sigma(0))$. Setting $a_{*}=\left(s_{\mathrm{in}}+x_{1}^{0}+\ldots+x_{n}^{0}\right) /(n+1)$, we can then take

$$
T_{2}(\ell)=T_{1}(\ell)+ \begin{cases}\left(\ell / a_{*}\right) T_{2}^{b}\left(2(n+1) a_{*}\right), & 0 \leq \ell \leq a_{*} \\ T_{2}^{b}\left((n+1)\left(\ell+a_{*}\right)\right), & \ell>a_{*}\end{cases}
$$

to satisfy our requirements.

We next fix any constant $\lambda_{2} \in(0,1)$ and set

$$
\underline{s}_{\lambda}=\lambda_{2} \min \left\{s_{*}, \frac{D s_{\text {in }}+\underline{d}_{0}}{D+\lambda_{1} C \sum_{i=1}^{n} \frac{m_{i}}{a_{i}}}\right\}
$$

and $\underline{x}_{i \lambda}=\lambda_{2} \min \left\{x_{i *}, x_{i}^{0}+\left(\underline{d}_{i} / D\right)\right\}$ for all $i$ and prove:

Lemma 3: If Assumptions 1-3 hold, then there is a function $T_{3} \in \mathcal{K}_{\infty}$ such that $s(t) \geq \underline{s}_{\lambda}$ and $x_{i} \geq \underline{x}_{i \lambda}$ hold for all $t \geq T_{3}(|\mathcal{E}(0)|)$ and all $i \in \mathcal{P}$.

Proof: (Sketch.) For all $t \geq T_{2}(|\mathcal{E}(0)|)$, Lemma 2 gives $x_{i}(t) \leq \lambda_{1} C$ for all $i \in\{1,2, \ldots, n\}$. Hence, for all $t \geq$ $T_{2}(|\mathcal{E}(0)|)$, (3) and our formula (4) for the $\mu_{i}$ 's give

$$
\dot{s}(t) \geq D\left(s_{\text {in }}-s(t)\right)-\lambda_{1} C \sum_{i=1}^{n} \frac{m_{i} s(t)}{a_{i}}+\underline{d}_{0}
$$

and

$$
\dot{x}_{i}(t) \geq-D x_{i}(t)+D x_{i}^{0}+\underline{d}_{i} \text { for all } i \in \mathcal{P} .
$$

The right side of (13) is bounded below by $\left(D s_{\text {in }}+\underline{d}_{0}\right)(1-$ $\left.\lambda_{2}\right)>0$ if $t$ is such that $s(t) \leq \underline{s}_{\lambda}$. Also, for each $i \in \mathcal{P}$, the right side of (14) is bounded below by $\left(1-\lambda_{2}\right)\left(D x_{i}^{0}+\underline{d}_{i}\right)>$ 0 if $t$ is such that $x_{i}(t) \leq \underline{x}_{i \lambda}$. Hence, for each $t_{0} \geq 0$ such that $s\left(t_{0}\right) \geq \underline{s}_{\lambda}$, we have $s(t) \geq \underline{s}_{\lambda}$ for all $t \geq t_{0}$; and for each $i \in \mathcal{P}$, and for each $t_{0} \geq 0$ such that $x_{i}\left(t_{0}\right) \geq \underline{x}_{i \lambda}$, we have $x_{i}(t) \geq \underline{x}_{i \lambda}$ for all $t \geq t_{0}$. Therefore', it suffices to choose $T_{3} \in \mathcal{K}_{\infty}$ such that: (i) If $s(0)<\underline{s}_{\lambda}$, then $s(t) \geq \underline{s}_{\lambda}$ for some $t \in\left[0, T_{3}(|\mathcal{E}(0)|)\right]$ (which implies that $s(t) \geq \underline{s}_{\lambda}$ for all $t \geq T_{3}(|\mathcal{E}(0)|)$, by the preceding argument) and (ii) for each $i \in \mathcal{P}$ such that $x_{i}(0)<\underline{x}_{i \lambda}$, we have $x_{i}(t) \geq \underline{x}_{i \lambda}$ for some $t \in\left[0, T_{3}(|\mathcal{E}(0)|)\right]$ (which implies that $x_{i}(t) \geq \underline{x}_{i \lambda}$ for all $t \geq T_{3}(|\mathcal{E}(0)|)$, also by the preceding argument).

To find $T_{3} \in \mathcal{K}_{\infty}$, first note that if we pick any constant

$$
T_{L}>\frac{1}{1-\lambda_{2}} \max \left\{\frac{\underline{s}_{\lambda}}{D s_{\text {in }}+\underline{d}_{0}}, \max \left\{\frac{\underline{x}_{i \lambda}}{D x_{i}^{0}+\underline{d}_{i}}: i \in \mathcal{P}\right\}\right\}
$$

then the Fundamental Theorem of Calculus and the positiveness of $s(t)$ and the $x_{i}(t)$ 's imply that: (A) If $s(0)<\underline{s}_{\lambda}$, then $s(\ell) \geq \underline{s}_{\lambda}$ for some $\ell \in\left[0, T_{L}\right]$, hence for all $\ell \geq T_{L}$ and (B) if $i \in \mathcal{P}$ is such that $x_{i}(0)<\underline{x}_{i \lambda}$, then $x_{i}(\ell) \geq \underline{x}_{i \lambda}$ for some $\ell \in\left[0, T_{L}\right]$, hence for all $\ell \geq T_{L}$. Conditions (A)(B) follow because $s(\ell) \geq s(0)+\ell\left(D s_{\text {in }}+\underline{d}_{0}\right)\left(1-\lambda_{2}\right)$ for all $\ell$ such that $\max _{r \in[0, \ell]} s(r)<\underline{s}_{\lambda}$, and $x_{i}(\ell) \geq x_{i}(0)+$ $\ell\left(1-\lambda_{2}\right)\left(D x_{i}^{0}+\underline{d}_{i}\right)$ for all $\ell$ such that $\max _{r \in[0, \ell]} x_{i}(r)<$ $\underline{x}_{i \lambda}$, so $\ell \leq T_{L}$. On the other hand, if $|\mathcal{E}(0)| \leq T_{M}$ where $T_{M}=\left(1-\lambda_{2}\right) \min \left\{s_{*}, \min \left\{x_{i *}: i \in \mathcal{P}\right\}\right\}$, then $s(0)-s_{*} \geq-|\mathcal{E}(0)| \geq\left(\lambda_{2}-1\right) s_{*}$; and for all $i \in \mathcal{P}$, we have $x_{i}(0)-x_{i *} \geq-|\mathcal{E}(0)| \geq\left(\lambda_{2}-1\right) x_{i *}$; so $s(0) \geq \lambda_{2} s_{*} \geq \underline{s}_{\lambda}$ and $x_{i}(0) \geq \lambda_{2} x_{i *} \geq \underline{x}_{i \lambda}$ for all $i \in \mathcal{P}$, which imply that $s(t) \geq \underline{s}_{\lambda}$ and $x_{i}(t) \geq \underline{x}_{i \lambda}$ hold for all $t \geq 0$ and $i \in \mathcal{P}$, by the previous paragraph. Hence,

$$
T_{3}(r)=T_{2}(r)+ \begin{cases}\left(T_{L}+T_{M}\right) \frac{r}{T_{M}}, & 0 \leq r \leq T_{M} \\ T_{L}+r, & r>T_{M}\end{cases}
$$

satisfies our requirements.

\section{B. Representing the Error Dynamics}

Let us introduce the functions and the constant

$$
\begin{aligned}
& \Gamma(s)=D+\sum_{i=1}^{n} \frac{a_{i} m_{i} x_{i *}}{\left(a_{i}+s_{*}\right)\left(a_{i}+s\right)}, \quad \tilde{s}(t)=s(t)-s_{*}, \\
& \tilde{x}_{i}(t)=x_{i}(t)-x_{i *} \text { for } i=1,2, \ldots, n, \\
& \text { and } \Gamma_{0}=D+\sum_{i=1}^{n} \frac{a_{i} m_{i} x_{i *}}{\left(a_{i}+s_{*}\right)\left(a_{i}+\bar{s}^{\sharp}\right)} .
\end{aligned}
$$


Then $\Gamma(s(t)) \geq \Gamma_{0}$ for all $t \geq 0$. By our formulas for $s_{\text {in }}$ and the $x_{i *}$ 's from (5) and (6), we have $D s_{\text {in }}=D s_{*}+$ $\mu_{1}\left(s_{*}\right) x_{1 *}+\ldots+\mu_{n}\left(s_{*}\right) x_{n *}$ and $D x_{i}^{0}=D x_{i *}-\mu_{i}\left(s_{*}\right) x_{i *}$ for all $i \in\{1,2, \ldots, n\}$. Hence, using (3) and the formulas for $x_{i *}$ from (6), and reorganizing terms gives:

$$
\left\{\begin{aligned}
\dot{\tilde{s}}(t)= & -D \tilde{s}(t)+\sum_{i=1}^{n}\left[\mu_{i}\left(s_{*}\right)-\mu_{i}(s(t))\right] x_{i *} \\
& -\sum_{i=1}^{n} \mu_{i}(s(t)) \tilde{x}_{i}(t)+\delta_{0}(t) \\
\dot{\tilde{x}}_{i}(t)= & {\left[\mu_{i}(s(t))-\mu_{i}\left(s_{*}\right)\right] x_{i}(t)+\tilde{x}_{i}(t) \mu_{i}\left(s_{*}\right) } \\
& -D \tilde{x}_{i}(t)+\delta_{i}(t), i=1, \ldots, n .
\end{aligned}\right.
$$

For all $s>0$, we can use our formulas (4) for the $\mu_{i}$ 's to check that $\Gamma$ can be rewritten as

$$
\Gamma(s)=D+\sum_{i=1}^{n} \frac{\mu_{i}(s)-\mu_{i}\left(s_{*}\right)}{s-s_{*}} x_{i *}
$$

for all $s \neq s_{*}$. Using the constants $p_{i}=D-\mu_{i}\left(s_{*}\right)$ (which are positive, by (5) in Assumption 2), we obtain

$$
\left\{\begin{aligned}
\dot{\tilde{s}}(t)= & -\Gamma(s(t)) \tilde{s}(t)-\sum_{i=1}^{n} \mu_{i}(s(t)) \tilde{x}_{i}(t)+\delta_{0}(t) \\
\dot{\tilde{x}}_{i}(t)= & -p_{i} \tilde{x}_{i}(t)+c_{i} x_{i}(t) \mu_{i}(s(t)) \frac{\tilde{s}(t)}{s(t)} \\
& +\delta_{i}(t), i=1, \ldots, n,
\end{aligned}\right.
$$

where $c_{i}=\frac{a_{i}}{a_{i}+s_{*}}$ for all $i \in\{1, \ldots, n\}$.

\section{Construction of a Lyapunov-Like Functional}

Let us define the $C^{1}$ function $V$ by

$$
\begin{aligned}
& V(\mathcal{E})=\nu(\tilde{s})+\sum_{i=1}^{n} \frac{1}{c_{i}} \Psi_{i}\left(\tilde{x}_{i}\right), \text { where } \\
& \nu(\tilde{s})=\tilde{s}-s_{*} \ln \left(\frac{\tilde{s}+s_{*}}{s_{*}}\right) \\
& \text { and } \Psi_{i}\left(\tilde{x}_{i}\right)=\tilde{x}_{i}-x_{i *} \ln \left(\frac{\tilde{x}_{i}+x_{i *}}{x_{i *}}\right)
\end{aligned}
$$

for all $i \in \mathcal{P}$, and $\Psi_{i}\left(\tilde{x}_{i}\right)=x_{i}$ for all $i \in\{1, \ldots, n\} \backslash \mathcal{P}$. By the chain rule, it follows that for all $t \geq T_{3}(|\mathcal{E}(0)|)$, the time derivative of $V$ along the solutions of (19) satisfies

$$
\begin{aligned}
\dot{V}(t) & \leq-\mathcal{N}(\mathcal{E}(t))+\bar{N}|\delta|_{[0, t]}, \text { where } \\
\mathcal{N}(\mathcal{E}(t)) & =\Gamma_{0} \frac{\tilde{s}^{2}(t)}{s(t)}+\sum_{i=1}^{n} q_{i} \frac{\tilde{x}_{i}^{2}(t)}{x_{i}(t)}
\end{aligned}
$$

and where $q_{i}=p_{i} / c_{i}=\left(D-\mu_{i}\left(s_{*}\right)\right) / c_{i}$ and the formula

$$
\bar{N}=(n+1) \max \left\{\frac{\bar{s}^{\sharp}+s_{*}}{\underline{s}_{\lambda}}, \max _{i \notin \mathcal{P}} \frac{1}{c_{i}}, \max _{i \in \mathcal{P}} \frac{\lambda_{1} C+x_{i *}}{c_{i} \underline{x}_{\lambda}}\right\}
$$

follows from Lemmas 2-3. Although (20) only holds for times $t \geq T_{3}(|\mathcal{E}(0)|)$, we can combine (20) with Gronwall's inequality to produce the final ISS estimate; see the appendix.

\section{ILLUSTRATION}

Consider the system (3) with $n=2, D=0.4, s_{*}=0.5$, $x_{1}^{0}=1$, and $x_{2}^{0}=0.55$ and the growth functions

$$
\mu_{1}(s)=\frac{s}{5+s} \quad \text { and } \quad \mu_{2}(s)=\frac{s}{2+s} .
$$

Then Assumption 2 is satisfied with $s_{\text {in }}=1.34412$, and our formulas (6) for the $x_{i *}$ 's give

$$
x_{1 *}=1.29412 \text { and } x_{2 *}=1.1 .
$$

In this illustration, we will only use the uncertainty vectors $\delta(t)$ to model uncertainties in applying the constant input concentrations $x_{1}^{0}$ and $x_{2}^{0}$ (which may occur in applications, because it may be difficult to maintain the inputs $x_{i}^{0}$ at constant levels), so we set $\delta_{0}(t)=0$ and therefore can choose $\underline{d}_{0}=\bar{d}_{0}=0, \underline{d}_{1}=-0.39, \underline{d}_{2}=-0.21$, and any constants $\bar{d}_{1} \geq 0$ and $\bar{d}_{2} \geq 0$ to satisfy all of our assumptions.

We simulated (3) using the command NDSolve in Mathematica, with the preceding choices of the parameters, and the disturbance vector $\delta(t)=\left(\delta_{0}(t), \delta_{1}(t), \delta_{2}(t)\right)=$ $(0,-0.1 \sin (t), 0.1 \cos (t))$. We report our results in Fig. 1, with the initial state $\left(s(0), x_{1}(0), x_{2}(0)\right)=(0.2,0.1,1)$, and then with the initial state $\left(s(0), x_{1}(0), x_{2}(0)\right)=$ $(1.3,0.2,0.1)$. The figure shows rapid convergence towards an oscillatory steady state, with a deviation from the equilibrium $\left(s_{*}, x_{1 *}, x_{2 *}\right)=(0.5,1.29412,1.1)$ that can be explained by the presence of the uncertainties $\delta_{1}$ and $\delta_{2}$, and therefore helps illustrate our theory.
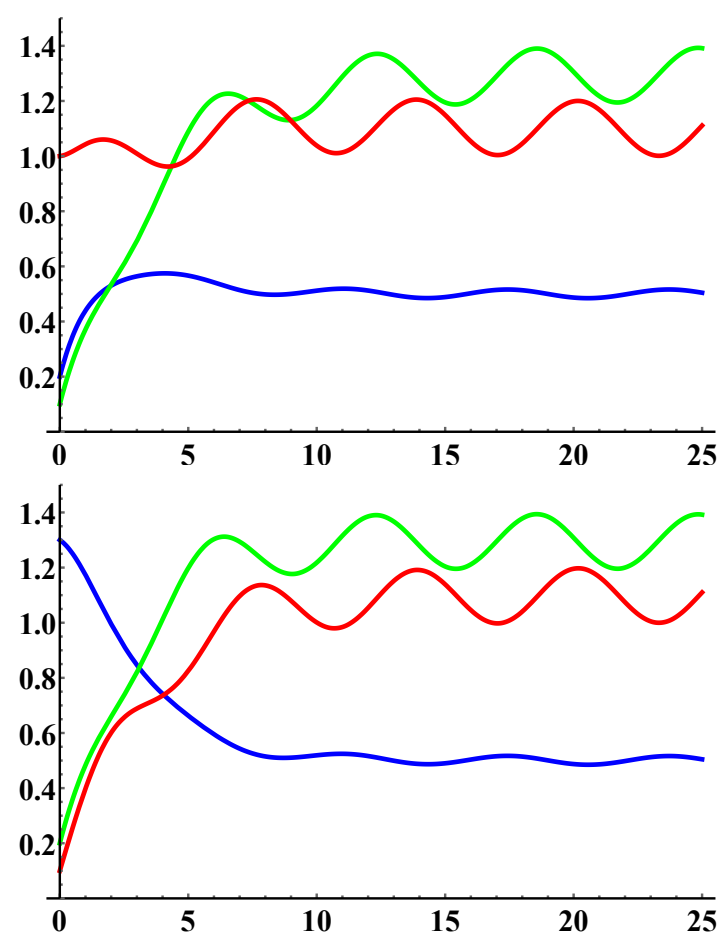

Fig. 1. Solution Components of (3) on Time Interval [0,25]. Species $x_{1}(t)$ and $x_{2}(t)$ are Green and Red Curves, Respectively. Substrate $s(t)$ is Blue Curve. Top Panel: Using Initial State $\left(s(0), x_{1}(0), x_{2}(0)\right)=(0.2,0.1,1)$. Bottom Panel: Using Initial State $\left(s(0), x_{1}(0), x_{2}(0)\right)=(1.3,0.2,0.1)$.

\section{CONCLUSIONS}

We solved a key input-to-state stabilization problem for a chemostat model with one limiting substrate, an arbitrary number of competing species, a constant dilution rate, and uncertainties, using constant inputs of the species. In the special case where the uncertainties are zero, this implies that all solutions with initial states in $(0, \infty)^{n+1}$ remain in $(0, \infty)^{n+1}$ at all future times and asymptotically converge to an equilibrium, which corresponds to persistence of all species whose constant inputs are positive. This contrasts with the competitive exclusion principle, which does not consider 
the possibility of introducing positive constant inputs of the species. The uncertainties can represent unmodeled features that commonly occur in biotechnological applications, so our work has the potential to benefit the study of some robustness issues.

\section{ApPendiX: LAST PART OF PROOF OF THEOREM 1}

To convert (20) into an ISS estimate for all $t \geq 0$, first note that we can use our lemmas to find a $\gamma \in \mathcal{K}_{\infty}$ such that

$$
\Gamma_{0} \frac{\tilde{s}^{2}(t)}{s(t)}+\sum_{i=1}^{n} q_{i} \frac{\tilde{x}_{i}^{2}(t)}{x_{i}(t)} \geq \underline{\gamma}(V(\mathcal{E}(t)))
$$

and therefore also

$$
\frac{d}{d t} V(\mathcal{E}(t)) \leq-\underline{\gamma}(V(\mathcal{E}(t)))+\bar{N}|\delta|_{[0, t]}
$$

along all trajectories of the $\mathcal{E}$ dynamics starting in our set $\mathcal{S}$ of initial states from the statement of our theorem, and for all $t \geq T_{3}(|\mathcal{E}(0)|)$. One method for finding $\underline{\gamma}$ is as follows. First, let $\mathcal{O}$ be the set of all points $\left(\tilde{s}, \tilde{x}_{1}, \ldots \tilde{x}_{n}\right) \in \mathbb{R}^{n+1}$ such that (i) $\tilde{s} \in\left[\underline{s}_{\lambda}-s_{*}, \lambda_{1} C-s_{*}\right]$, (ii) $\tilde{x}_{i} \in\left[\underline{x}_{i \lambda}-x_{i *}, \lambda_{1} C-x_{i *}\right]$ for all $i \in \mathcal{P}$, and (iii) $\tilde{x} \in\left(0, \lambda_{1} C\right]$ for all $i \in\{1, \ldots, n\} \backslash \mathcal{P}$. Next, pick a function $\underline{\gamma}_{0} \in \mathcal{K}_{\infty}$ such that

$$
(n+1) \nu(\tilde{s}) \leq \underline{\gamma}_{0}(|\tilde{s}|) \text { and }(n+1) \frac{\Psi_{i}\left(\tilde{x}_{i}\right)}{c_{i}} \leq \underline{\gamma}_{0}\left(\left|\tilde{x}_{i}\right|\right)
$$

for all values $\mathcal{E} \in \mathcal{O}$. Then

$$
V_{1}(\mathcal{E}(t)) \leq \underline{\gamma}_{0}(|\mathcal{E}(t)|)
$$

for all $t \geq T_{3}(|\mathcal{E}(0)|)$. Hence, we can use Lemma 2 to obtain

$$
\left(\underline{\gamma}_{0}^{-1}\left(V_{1}(\mathcal{E}(t))\right)\right)^{2} \leq|\mathcal{E}(t)|^{2} \leq \lambda_{1} C\left(\frac{\tilde{s}^{2}(t)}{s(t)}+\sum_{i=1}^{n} \frac{\tilde{x}_{i}^{2}(t)}{x_{i}(t)}\right)
$$

for all $t \geq T_{3}(|\mathcal{E}(0)|)$. Therefore, we can choose

$$
\underline{\gamma}(r)=\frac{1}{\lambda_{1} C}\left(\underline{\gamma}_{0}^{-1}(r)\right)^{2} \min \left\{\Gamma_{0}, q_{1}, \ldots, q_{n}\right\} .
$$

Hence, combining (20) and (A.1) gives (A.2), so standard ISS arguments [6] provide $\beta_{0} \in \mathcal{K} \mathcal{L}$ and $\gamma_{0} \in \mathcal{K}_{\infty}$ such that

$$
V(\mathcal{E}(t)) \leq \beta_{0}\left(V\left(\mathcal{E}\left(T_{3}(|\mathcal{E}(0)|)\right)\right), t\right)+\gamma_{0}\left(|\delta|_{[0, t]}\right)
$$

holds for all $t \geq T_{3}(|\mathcal{E}(0)|)$. Then the structure of $V$ provides functions $\beta_{1} \in \mathcal{K} \mathcal{L}$ and $\gamma_{1} \in \mathcal{K}_{\infty}$ such that

$$
|\mathcal{E}(t)| \leq \beta_{1}\left(\left|\mathcal{E}\left(T_{3}(|\mathcal{E}(0)|)\right)\right|, t\right)+\gamma_{1}\left(|\delta|_{\infty}\right)
$$

for all $t \geq T_{3}(|\mathcal{E}(0)|)$.

To extend (A.6) to obtain an ISS estimate on $[0,+\infty)$, first note that the structure of the $\mathcal{E}$ dynamics (17), combined with our bounds on the $\mu_{i}$ 's and $\Gamma$ and the Lipschitzness of the $\mu_{i}$ 's, provide a constant $\bar{L}$ (that is independent of the choice of the solution) such that

$$
\left|\mathcal{E}^{\prime}(\ell)\right| \leq \bar{L}\left(|\mathcal{E}(\ell)|+|\delta|_{\infty}\right)
$$

holds for all $\ell \in\left[0, T_{3}(|\mathcal{E}(0)|)\right]$; this can be done by rewriting $x_{i}(t)$ in (17) in the form $\tilde{x}_{i}(t)+x_{i *}$. Integrating (A.7) over $[0, t]$ for any $t \in\left[0, T_{3}(|\mathcal{E}(0)|)\right]$ and applying the Fundamental Theorem of Calculus to $\mathcal{E}$ gives

$$
|\mathcal{E}(t)| \leq|\mathcal{E}(0)|+\bar{L} \int_{0}^{t}|\mathcal{E}(\ell)| \mathrm{d} \ell+\bar{L} T_{3}(|\mathcal{E}(0)|)|\delta|_{\infty}
$$

We now apply Gronwall's inequality [6] to $|\mathcal{E}|$ to get

$$
\begin{aligned}
|\mathcal{E}(t)| \leq & |\mathcal{E}(0)| e^{\bar{L} T_{3}(|\mathcal{E}(0)|)} \\
& +\left\{\bar{L} T_{3}(|\mathcal{E}(0)|) e^{\bar{L} T_{3}(|\mathcal{E}(0)|)}\right\}\left\{|\delta|_{\infty}\right\} \\
\leq & e^{T_{3}(|\mathcal{E}(0)|)-t}\left[|\mathcal{E}(0)| e^{\bar{L} T_{3}(|\mathcal{E}(0)|)}\right. \\
& \left.+\frac{1}{2} \bar{L}^{2} T_{3}^{2}(|\mathcal{E}(0)|) e^{2 \bar{L} T_{3}(|\mathcal{E}(0)|)}\right]+\frac{1}{2}|\delta|_{\infty}^{2}
\end{aligned}
$$

for all $t \in\left[0, T_{3}(|\mathcal{E}(0)|)\right]$, by applying the triangle inequality to the terms in curly braces in (A.9). The ISS estimate now follows from adding the bounds (A.6) and (A.9), and using (A.9) with the choice $t=T_{3}(|\mathcal{E}(0)|)$ to upper bound the $\left|\mathcal{E}\left(T_{3}(|\mathcal{E}(0)|)\right)\right|$ that occurs in the right side of (A.6), because $|\mathcal{E}(t)|$ is independent of values of $\delta(r)$ for times $r>t$.

\section{REFERENCES}

[1] S. Dikshitulu, B. Baltzis, G. Lewandowski, and S. Pavlou. Competition between two microbial populations in a sequencing fed-batch reactor: theory, experimental verification, and implications for waste treatment applications. Biotechnology and Bioengineering, 42(5):643-656, 1993.

[2] J-L. Gouzé and G. Robledo. Feedback control for nonmonotone competition models in the chemostat. Nonlinear Analysis: Real World Applications, 6(4):671-690, 2005.

[3] F. Grognard, F. Mazenc, and A. Rapaport. Polytopic Lyapunov functions for persistence analysis of competing species. Discrete and Continuous Dynamical Systems Series B, 8(1):73-93, 2007.

[4] G. Hardin. Competitive exclusion principle. Science, 131(3409):12921297, 1960

[5] S-B. Hsu and P. Waltman. On a system of reaction-diffusion equations arising from competition in an unstirred chemostat. SIAM Journal on Applied Mathematics, 53(4):1026-1044, 1993.

[6] H. Khalil. Nonlinear Systems, Third Edition. Prentice-Hall, Englewood Cliffs, NJ, 2002.

[7] P. Lenas and S. Pavlou. Coexistence of three competing microbial populations in a chemostat with periodically varying dilution rate. Mathematical Biosciences, 129(2):111-142, 1995.

[8] C. Lobry, F. Mazenc, and A. Rapaport. Persistence in ecological models of competition for a single resource. Comptes Rendus Mathematique, 340(3):199-204, 2005.

[9] F. Mazenc, J. Harmand, and M. Malisoff. Stabilization in a chemostat with sampled and delayed measurements. In Proceedings of the American Control Conference (Boston, MA, 6-8 July 2016), pp. 18571862.

[10] F. Mazenc and Z-P. Jiang. Global output feedback stabilization of a chemostat with an arbitrary number of species. IEEE Transactions on Automatic Control, 55(11):2570-2575, 2010.

[11] F. Mazenc and M. Malisoff. Stabilization of a chemostat model with Haldane growth functions and a delay in the measurement. Automatica, 46(9):1428-1436, 2010.

[12] F. Mazenc and M. Malisoff. Stability and stabilization for models of chemostats with multiple limiting substrates. Journal of Biological Dynamics, 6(2):612-627, 2012.

[13] F. Mazenc, M. Malisoff, and J. Harmand. Further results on stabilization of periodic trajectories for a chemostat with two species. IEEE Transactions on Automatic Control, 53(Special Issue on Systems Biology):66-74, 2008.

[14] J. Monod. La technique de culture continue, théorie et applications. Annales de l'Institut Pasteur, 79:390-410, 1950.

[15] H. Nie and J. Wu. Coexistence of an unstirred chemostat model with Beddington-De Angelis functional response and inhibitor. Nonlinear Analysis: Real World Applications, 11(5):3639-3652, 2010.

[16] A. Novick and L. Szilard. Description of the chemostat. Science, 112(2920):715-716, 1950.

[17] G. Robledo, F. Grognard, and J-L. Gouzé. Global stability for a model of competition in the chemostat with microbial inputs. Nonlinear Analysis: Real World Applications, 13(2):582-598, 2012.

[18] H. Smith and P. Waltman. The Theory of the Chemostat. Dynamics of Microbial Competition. Cambridge University Press, Cambridge, UK, 1995.

[19] G. Wolkowicz and X-Q. Zhao. n-species competition in a periodic chemostat. Differential Integral Equations, 11(3):465-491, 1998. 